\title{
Conocimientos y Actitudes Asociados al Cuidado de la Salud Sexual y Reproductiva en los Adultos Jóvenes de la Ciudad de Panamá Durante el Año 2021
}

\author{
Centeno, Mariel \\ Universidad Católica Santa María la Antigua \\ Panamá, Panamá \\ mcentenos@usma.com.pa \\ Saldaña, Paola \\ Universidad Católica Santa María la Antigua \\ Panamá, Panamá \\ psaldanaa@usma.com.pa
}

Oviedo, Diana

Universidad Católica Santa María la Antigua, Centro de Neurociencias y Unidad de Investigación Clínica, INDICASAT AIP, Sistema Nacional de Investigación (SNI), SENACYT

Panamá, Panamá

doviedoc@usma.com.pa

\section{Abstract}

Young people are the population most exposed to risky sexual behaviors such as: early initiation of sexual activity, promiscuity, unprotected intercourse, among others. The objective of this preliminary project is to determine the level of knowledge and attitudes associated with sexual and reproductive health care in young adults in Panama. This is a descriptive, quantitative, non-experimental, cross-sectional study. For this, 30 young people between 19 and 30 years old filled out the following questionnaires: The Questionnaire of Knowledge and Attitudes About Sexual Health and Reproductive Health, the Questionnaire of Sexual Risk Behavior in Young People, the Questionnaire of Attitudes About Prevention of Transmitted Infections Sexual and Contraceptive Methods and the Questionnaire of Attitudes about Risky Sexual Behaviors. Young adults presented a high level of knowledge about sexual and reproductive health (97\%) and, to a lesser extent (3\%), a medium level of knowledge on this subject. Young adults showed a neutral attitude about risky sexual behaviors (77\%) and, to a lesser extent (23\%), a favorable attitude on this topic. In conclusion, the majority of young adults present a high level of knowledge about sexual and reproductive health, as well as a neutral attitude towards risky sexual behaviors, although 
the group of believers practicing a religion is soon the group with the highest attitudes of risky sexual behavior. This may be due to the taboo that exists in Panama around sexuality, resulting in the lack of sexual education.

Keywords: Sexual Health, Risky Sexual Behaviors, Attitudes, Sexuality, Reproductive Health Care.

\section{Resumen}

Los jóvenes son la población más expuesta a conductas sexuales de riesgo como: el comienzo precoz de la actividad sexual, promiscuidad, coito sin protección, entre otros. El objetivo de este anteproyecto es determinar el nivel de conocimiento y las actitudes asociadas al cuidado de la salud sexual y reproductiva en los adultos jóvenes de Panamá. Este es un estudio descriptivo, cuantitativo, no experimental, transversal. Para ello, 30 jóvenes entre 19 a 30 años rellenaron los siguientes cuestionarios: El Cuestionario de Conocimientos y Actitudes Sobre la Salud Sexual y la Salud Reproductiva, el Cuestionario de Conducta Sexual de Riesgo en Jóvenes, el Cuestionario de Actitudes Sobre Prevención de Infecciones de Transmisión Sexual y Métodos Anticonceptivos y el Cuestionario de Actitudes sobre las Conductas Sexuales de Riesgo. Los adultos jóvenes presentaron un nivel alto en conocimientos sobre la salud sexual y reproductiva (97\%) y, en menor proporción (3\%) un nivel de conocimiento medio en dicha temática. Los adultos jóvenes presentaron una actitud neutral sobre las conductas sexuales riesgo (77\%) y, en menor proporción (23\%) una actitud favorable en dicha temática. En conclusión, la mayoría de los adultos jóvenes presentan un nivel alto de conocimientos sobre salud sexual y reproductiva, así como, una actitud neutral hacia las conductas sexuales de riesgo, aunque el colectivo de creyentes practicantes de una religión constituyó el grupo con mayores actitudes de conducta sexual de riesgo. Esto puede deberse al tabú que existe en Panamá alrededor de la sexualidad, trayendo como consecuencia la falta de educación sexual integral.

Palabras claves: Salud Sexual, Conductas Sexuales de Riesgo, Actitudes, Sexualidad, Salud Reproductiva.

\section{INTRODUCCIÓN}

La salud sexual sigue siendo un desafío en Panamá, y las estadísticas reflejan este grave problema. El 27,2\% de las adolescentes panameñas de entre 15 y 19 años tuvieron relaciones sexuales antes de los 15 años. El mismo colectivo tuvo al menos un embarazo 
durante este periodo de 4 años, según la Encuesta Nacional de Salud Sexual y Reproductiva de Panamá (2014-2015). Esto nos refleja que los jóvenes son la población más expuesta a conductas sexuales de riesgo [1].

Para abordar esta problemática se necesita que la población disponga de información clara y precisa sobre cómo evitar dichas conductas y así tomar decisiones más acertadas respecto a su salud sexual y reproductiva. Por ejemplo, se ha demostrado que la utilización de anticonceptivos es más constante si esta información se recibe antes de la primera relación sexual [1]. Además, la disposición de dichos conocimientos limita el inicio precoz de las relaciones sexuales [2]. Estudios realizados por García Madrid et al., [3] y Rojas Betancur et al., [4] sobre esta temática, señalan que existe una asociación significativa de los conocimientos que se tienen sobre la salud sexual, reproductiva, las actitudes y prácticas sexuales de los jóvenes. Por lo que, se puede considerar que esta problemática tiene su origen en el bajo nivel de conocimiento acerca del sexo y la sexualidad, lo cual conlleva en muchos casos a exhibir actitudes poco favorables frente a este aspecto.

\section{MÉTODO}

\section{A. PARTICIPANTES}

La población estaba compuesta por jóvenes adultos de 19-30 años de la ciudad de Panamá. Fue una muestra por conveniencia, no aleatoria, ya que se incluyeron todos los elementos necesarios y convenientes para esta investigación. El tamaño de la muestra fue de 30 adultos jóvenes, los cuales debían haber recibido mínimo educación primaria y haber tenido por lo menos un tipo de relación sexual.

\section{B. INSTRUMENTOS}

Para recoger los datos sociodemográficos se elaboró una encuesta sociodemográfica para recabar las variables independientes mencionadas anteriormente. La variables encontradas fueron: identidad de género, orientación sexual, nivel socioeconómico, nivel de educación, religión y profesión. Para evaluar los conocimientos y actitudes sobre salud sexual y reproductiva se utilizaron tres cuestionarios y una ficha de registro validados:

Cuestionario "Conocimientos y actitudes sobre la salud sexual y la salud reproductiva" [5], el cual mide el nivel de conocimiento sobre el cuidado de la salud sexual y reproductiva. Está dividido en 4 dimensiones: salud sexual, salud reproductiva, VIH/SIDA/ITS y uso de condón.

Ficha de registro "Conducta sexual de riesgo en jóvenes" [6], la cual identifica las conductas sexuales de riesgo: inicio de relaciones sexuales, número de parejas sexuales, aborto, uso de métodos anticonceptivos, relaciones sexuales en estado de intoxicación y 
revisiones periódicas ginecológicas y urológicas.

Cuestionario "Actitudes sobre prevención de infecciones de transmisión sexual y métodos anticonceptivos" [7], que pretende descubrir las actitudes que tienen los adultos jóvenes en cuanto a la prevención de infecciones de transmisión y métodos anticonceptivos.

Cuestionario "Actitudes sobre las conductas sexuales de riesgo" [8], que pretende descubrir las actitudes que tienen los adultos jóvenes en cuanto a las conductas sexuales de riesgo.

\section{PROCEDIMIENTO}

Los cuestionarios que conforman esta investigación se aplicaron en línea a través de la aplicación "Google Encuestas". Estos se difundieron por medio de redes sociales para iniciar el reclutamiento de los participantes. A través del formulario de Google se les pidió el consentimiento informado.

\section{RESULTADOS}

$70 \%$ de la muestra era solo mujer y $30 \%$ hombre. La mayoría se identificó como persona cisgenero (93.3\%), con orientación sexual heterosexual $(66.7 \%)$, cursando o concluida la Licenciatura (80\%), y con un nivel socioeconómico alto (53.4\%). En cuanto a religión, $60 \%$ de la muestra indicaron pertenecer a alguna religión; 33.3\% era creyente practicante. Con respecto al campo profesional, $20 \%$ pertenecía a las Ciencias Económicas y Administrativas, $20 \%$ a las Ciencias Exactas e Ingenierías, $36.7 \%$ a las Ciencias de la Salud y $23.3 \%$ a otras profesiones.

Para examinar las variables sociodemográficas se utilizaron análisis descriptivos. Los resultados se presentan en frecuencia (F), medias (M) y desviación estándar (DE). Posteriormente, se realizaron ANOVAS para comparar entre los grupos de interés: nivel de religiosidad, orientación sexual y campo profesional.

En relación con el nivel de religiosidad, sujetos pertenecientes al grupo no creyentes denotaron actitudes de menor conducta sexual de riesgo, puesto que, obtuvieron una puntuación media más alta $(\mathrm{M}=54.3, \mathrm{DE}=4.03)$ en la escala de actitudes hacia las conductas sexuales de riesgo, en comparación con el grupo de creyentes practicantes ( $M=46.4$, $\mathrm{DE}=4.84)$ y creyentes no practicantes $(\mathrm{M}=49, \mathrm{DE}=7.25)$, siendo la diferencia estadísticamente significativa, $\mathrm{F}=8.54, \mathrm{p}=0.003$ (tabla 1 ). 
Tabla 1. Conocimientos y actitudes hacia la Salud Sexual y Reproductiva según nivel de religión

\begin{tabular}{cccccc}
\hline Variable & $\begin{array}{c}\text { Creyente Practicante } \\
(n=10)\end{array}$ & $\begin{array}{c}\text { Creyente No Practicante } \\
(n=8)\end{array}$ & $\begin{array}{c}\text { No Creyente }(n=12) \\
\text { Media }(D E)\end{array}$ & $F$ & $p$ \\
& Media (DE) & Media (DE) & & & \\
\hline DITS & $42,8(16,19)$ & $44,9(10,51)$ & $35,5(8,50)$ & 2,44 & 0,121 \\
PtotalSSySR & $124,2(27,89)$ & $124,4(23,27)$ & $103,5(17,27)$ & 3,39 & 0,061 \\
ActitudeSCSR & $46,4(4,84)$ & $49(7,25)$ & $54,3(4,03)$ & 8,54 & 0,003 \\
\hline
\end{tabular}

Nota DSS: Conocimientos sobre salud sexual; DSR: Conocimientos sobre salud reproductiva; PtotalSSySR: Conocimientos sobre salud sexual y reproductiva; ActitudesCSR: Puntuación total de la escala de actitudes hacia las conductas sexuales de riesgo.

Con respecto a la orientación sexual, se encontraron diferencias significativas $(p<0.05)$ entre el grupo LGBTQ+ y el grupo de heterosexuales. Los sujetos pertenecientes al grupo de LGBTQ+ denotaron un conocimiento más alto sobre salud sexual y reproductiva, ya que obtuvieron una puntuación media más baja ( $M=105.4, D E=15.28)$, en comparación con el grupo de heterosexuales $(M=121.3, D E=26.56)$, siendo la diferencia estadísticamente significativa, $F=4.28, p=0.048$. De igual forma, el grupo LGBTQ+ demostró actitudes de menor conducta sexual de riesgo, puesto que, obtuvieron una puntuación media más alta $(M=53.1, D E=2.18)$, frente al grupo de heterosexuales $(M=48.9, D E=2.18)$, comprendiendo una diferencia estadísticamente significativa, $\mathrm{F}=6.04, \mathrm{p}=0.021$ (Tabla 2).

\begin{tabular}{ccccc}
\hline Variable & $\begin{array}{c}\text { Heterosexual } \\
(n=20) \\
\text { Media (DE) }\end{array}$ & $\begin{array}{c}\text { LGBTQ+ } \\
(\mathrm{n}=10) \\
\text { Media (DE) }\end{array}$ & $\mathrm{F}$ & $\mathrm{p}$ \\
\hline DSS & $21,5(6,06)$ & $17,3(4,76)$ & 4,30 & 0,050 \\
DSR & $22,6(7,02)$ & $18,2(4,21)$ & 4,46 & 0,044 \\
PtotalSSySR & $121,3(26,56)$ & $105,4(15,28)$ & 4,28 & 0,048 \\
ActitudesCSR & $48,9(2,18)$ & $53,1(2,18)$ & 6,04 & 0,021 \\
\hline
\end{tabular}

Tabla 2. Conocimientos y actitudes hacia la Salud Sexual y Reproductiva según la orientación sexual

Con relación a la profesión, los sujetos pertenecientes a otros campos profesionales obtuvieron una puntuación más alta en cuanto a conocimientos sobre salud sexual ( $M=17.1$, $D=3.23)$, seguido de Ciencias de la Salud $(M=19.0, D E=5.23)$ y Ciencias Exactas y de la Ingeniería ( $M=20.8, D E=8.20)$, en comparación con el grupo concerniente a las Ciencias Económicas y Administrativas ( $M=25.5, D E=5.32)$, siendo la diferencia casi estadísticamente significativa, $\mathrm{F}=3.53, \mathrm{p}=0.052$. Por el contrario, el grupo comprendido por las Ciencias de la Salud obtuvo una puntuación más alta en los conocimientos sobre la salud reproductiva $(M=17.5, D E=4.01)$, seguido por el grupo de otros campos profesionales ( $M=18.8, D E=3.77)$, en comparación con el grupo de Ciencias Exactas y de la Ingeniería $(M=27.4, D E=4.98)$ y 
Ciencias Económicas y Administrativas ( $M=25.7, D E=8.36)$, comprendiendo una diferencia estadísticamente significativa, $\mathrm{F}=5.63, \mathrm{p}=0.014$ (Tabla 3).

Tabla 4. Conocimientos y actitudes hacia la Salud Sexual y Reproductiva según la profesión

\begin{tabular}{|c|c|c|c|c|c|c|}
\hline Variable & $\begin{array}{c}\text { Ciencias Económicas y } \\
\text { Administrativas } \\
(n=6) \\
\text { Media (DE) }\end{array}$ & $\begin{array}{c}\text { Ciencias de la } \\
\text { Salud } \\
(n=11) \\
\text { Media (DE) }\end{array}$ & $\begin{array}{l}\text { Ciencias Exactas y } \\
\text { de la Ingeniería } \\
(n=5) \\
\text { Media (DE) }\end{array}$ & $\begin{array}{c}\text { Otros Campos } \\
\text { Profesionales } \\
(n=8) \\
\text { Media (DE) }\end{array}$ & $\mathrm{F}$ & $\mathrm{p}$ \\
\hline DSS & $25,5(5,32)$ & $19,0(5.23)$ & $20,8(8,20)$ & $17,1(3,23)$ & 3,53 & \\
\hline DSR & $25,7(8,36)$ & $17,5(4.01)$ & $27,4(4,98)$ & $18,8(3,77)$ & 5,63 & \\
\hline PtotalSSySR & $141,2(30,97)$ & $108,1(17.87)$ & $123,6(21,52)$ & $103,1(13,29)$ & 3,07 & \\
\hline ActitudesCSR & $35,7(7,26)$ & $31,8(4.60)$ & $34,4(6,54)$ & $39,0(5,61)$ & 1,74 & \\
\hline
\end{tabular}

Los adultos jóvenes presentaron un nivel alto en conocimientos sobre la salud sexual y reproductiva (97\%) y, en menor proporción (3\%) un nivel de conocimiento medio en dicha temática (Figura 1).

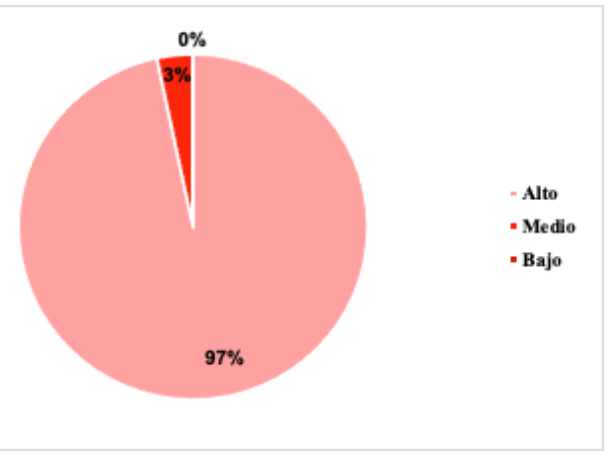

Figura 1. Nivel de conocimiento sobre la salud sexual y la salud reproductiva

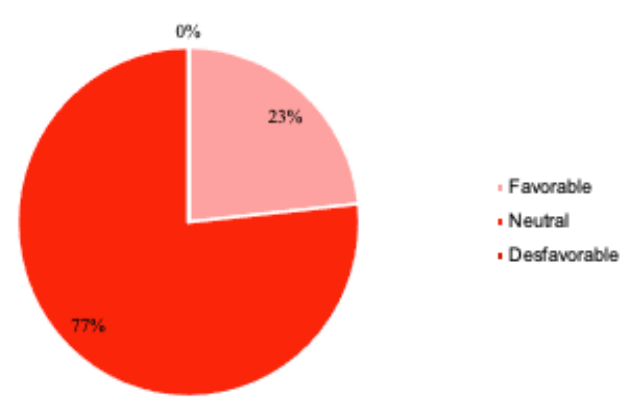

Figura 2. Actitudes sobre conductas sexuales de riesgo

Los adultos jóvenes presentaron una actitud neutral sobre las conductas sexuales riesgo (77\%) y, en menor proporción (23\%) una actitud favorable en dicha temática (Figura 2). 
Con respecto a conductas sexuales de riesgo, más de dos quintas partes de la muestra (46.66\%) han tenido relaciones sexuales en estado de intoxicación; en caso de embarazo un tercio (30\%) recurriría al aborto, aunque la única opción sea la insegura; un tercio (30\%) nunca ha asistido a revisiones médicas sexuales o reproductivas.

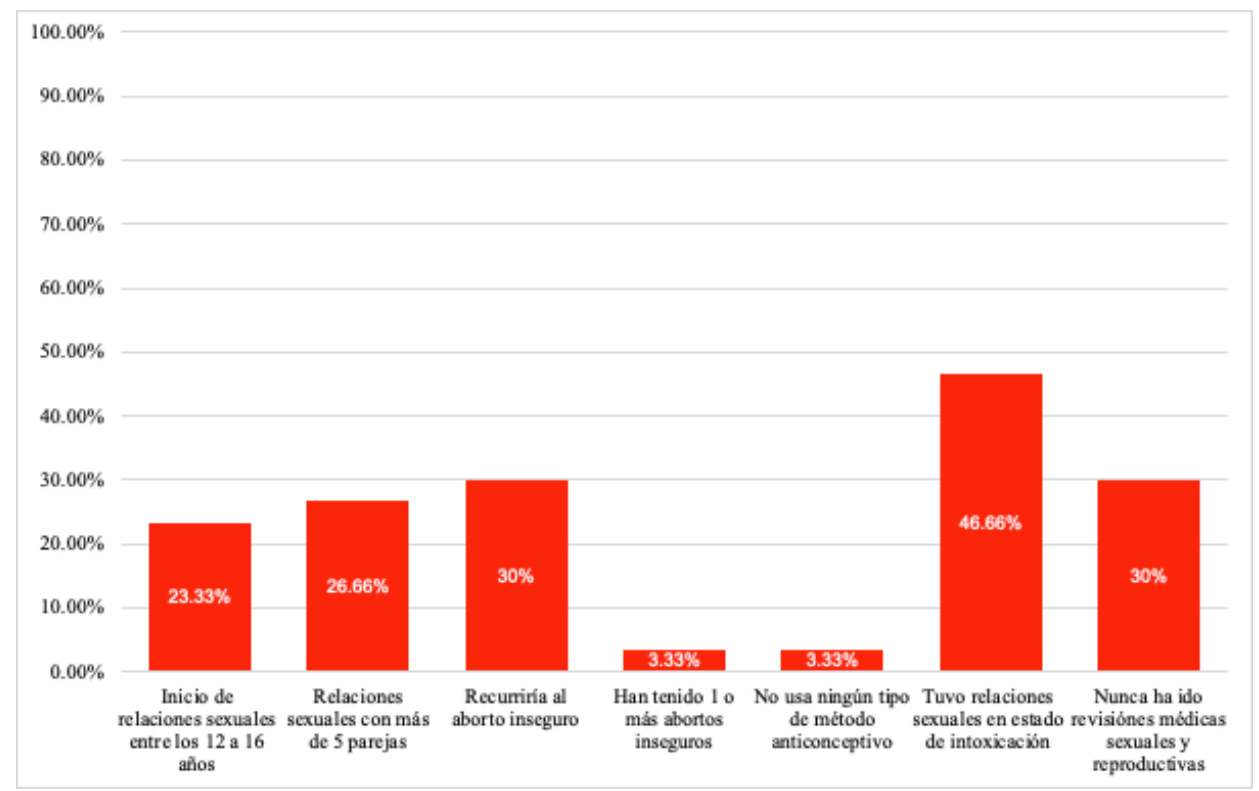

Figura 3. Conductas sexuales de riesgo de los adultos jóvenes

\section{CONCLUSIONES}

En Panamá, se encontró que la mayoría de los adultos jóvenes presentan un nivel alto de conocimiento sobre la salud sexual y reproductiva, una actitud favorable hacia la prevención de ITS y métodos anticonceptivos, así como, una actitud neutral hacia las conductas sexuales de riesgo. Al comparar los resultados con respecto a otros estudios, no se encontraron resultados similares. En el estudio por Ordoñez Sánchez et al., [9] se encontró que la mayoría de los estudiantes universitarios de 18-25 años tienen un nivel medio de conocimientos sobre salud sexual medio y una actitud desfavorable hacia las conductas sexuales de riesgo. Así mismo, el estudio de Paredes Ramirez, N., \& Chavez Rengifo, L. [6] demostró que, la mayoría de los adolescentes poseen un nivel medio de conocimientos sobre salud sexual y una actitud desfavorable hacia las conductas sexuales de riesgo.

Por otro lado, los conocimientos y actitudes menos favorables hacia la salud sexual y reproductiva, así como las conductas sexuales de riesgo, están relacionadas con las características sociodemográficas de nivel de religiosidad, orientación sexual y campo profesional, concluyendo que estos factores son los que impactan significativamente en 
la toma de decisiones concerniente al cuidado de la salud sexual y reproductiva. Dichas características sociodemográficas se encuentran específicamente en los grupos de sujetos creyentes practicantes, con orientación heterosexual, en el campo profesional de Ciencias Económicas y Administrativas.

En cuanto a las conductas sexuales de riesgo, se encontró que, parte de la muestra han tenido relaciones sexuales en estado de intoxicación, en caso de embarazo recurrirían al aborto, aunque la unica opción sea la insegura, y nunca ha asistido a revisiones médicas sexuales o reproductivas. Además, se observó que gran parte de los adultos jóvenes asisten con frecuencia a revisiones médicas y sexuales.

\section{Referencias}

[1] ENNASER, Encuesta Nacional de Salud Sexual y Reproductiva Panamá, 2014-2015, UNFPA, 2015.

[2] C. Quintana Pantaleón, «Sexualidad y anticoncepción en la adolescencia,» Pediatría Integral, vol. XVII, no 3, pp. 171-184, 2013.

[3] A. Ruiz García, Ó. Jiménez, M. A. Ojeda Mora, M. Á. I Rando Hurtado y L. Martínez Suárez, «Intervención grupal en educación sexual con estudiantes: actitudes hacia la sexualidad, masturbación y fantasías sexuales,» Escritos de Psicología, vol. 12, № 1, pp. 30-37, 2019.

[4] M. Rojas Betancur, R. Méndez Villamizar y L. Montero Torres, «Salud sexual y reproductiva en adolescentes: la fragilidad de la autonomía," Hacia la Promoción de la Salud, vol. 21, no 1, pp. 52 62, 2016.

[5] R. V. Ruiz, «La salud sexual y la salud reproductiva de la población estudiantil de la Universidad Nacional,» 2007.

[6] N. P. Ramirez y L. C. Rengifo, "Conocimiento sobre salud sexual y conductas sexuales de riesgo, en adolescentes del asentamiento poblacional Micaela Bastidas, Iquitos, 2015.

[7] C. R. Takahashi y A. M. V. Zevallos, «Conocimiento y actitudes sobre prevención de infecciones de transmisión sexual y métodos anticonceptivos en estudiantes universitarios en una carrera de salud de una universidad privada de Lima, 2017.

[8] V. M. A. Mejía, «Conocimientos y actitudes sobre el inicio de relaciones sexuales en adolescentes y comunicación familiar en instituciones educativas públicas - Hualmay 2015,» 2018.

[9] J. Ordoñez Sánchez, J. Real Cotto, J. Gallardo León, H. Alvarado Franco y A. Roby Arias, «Conocimientos sobre salud sexual y su relación con el comportamiento sexual en estudiantes universitarios," Anales de la Facultad de Medicina, vol. 78, no 4, p. 419, 2018.

\section{Autorización y Licencia CC}

Los autores autorizan a APANAC XVIII a publicar el artículo en las actas de la conferencia en Acceso Abierto (Open Access) en diversos formatos digitales (PDF, HTML, EPUB) e integrarlos en diversas plataformas online como repositorios y bases de datos bajo la licencia CC:

Attribution-NonCommercial-ShareAlike 4.0 International (CC BY-NC-SA 4.0) https://creativecommons. org/licenses/by-nc-sa/4.0/. Ni APANAC XVIII ni los editores son responsables ni del contenido ni de las implicaciones de lo expresado en el artículo. 